\title{
Integration of the Thiol Redox Status with Cytokine Response to Physical Training in Professional Basketball Players
}

\section{A. ZEMBRON-LACNY ${ }^{1}$, M. SLOWINSKA-LISOWSKA ${ }^{2}$, A. ZIEMBA ${ }^{3}$}

${ }^{1}$ Department of Biochemistry and Sports Medicine, Faculty of Physical Culture Gorzow Wlkp. University of Physical Education Poznan, Poland, ${ }^{2}$ Department of Sport Medicine, University of Physical Education Wroclaw, Poland, ${ }^{3}$ Department of Applied Physiology, Mossakowski Medical Research Center, Polish Academy of Sciences Warsaw, Poland

Received February 23, 2009

Accepted May 26, 2009

On-line June 19, 2009

\section{Summary}

The present study was designed to evaluate the plasma markers of reactive oxygen species (ROS) activity and cytokines, and their relationship with thiol redox status of basketball players during training. Sixteen professional players of the Polish Basketball Extraleague participated in the study. The study was performed during the preparatory period and the play-off round. Markers of ROS activity (lipid peroxidation TBARS, protein carbonylation PC) and reduced glutathione (GSH) demonstrated regularity over time, i.e. TBARS, PC and GSH were elevated at the beginning and decreased at the end of training periods. Oxidized glutathione (GSSG) was not affected by exercise training. Thiol redox status ( $\mathrm{GSH}_{\text {total }}-2 \mathrm{GSSG} / \mathrm{GSSG}$ ) correlated with TBARS and PC in both training periods. The level of interleukin- 6 (IL-6) was increased and positively correlated with thiol redox $(r=0.423)$ in the preparatory period, whereas tumor necrosis factor $\alpha$ (TNF $\alpha$ ) was increased and inversely correlated with thiol redox $(r=-0.509)$ in the play-off round. The present study showed significant shifts in markers of ROS activity, thiol redox status and inflammatory mediators (IL-6, TNF $\alpha$ ) following professional sport training as well as correlation between changes in thiol redox and cytokine response.

\section{Key words}

Cytokines - Thiol redox • Lipid peroxidation - Protein carbonylation • Athletes

\section{Corresponding author}

A. Zembron-Lacny, University of Physical Education Poznan, Faculty of Physical Culture Gorzow Wlkp., Department of Biochemistry, Estkowskiego 13, 66-400 Gorzow Wlkp., Poland. Fax: 004895 7279231. E-mail: agzem@gorzow.home.pl

\section{Introduction}

The induction of reactive oxygen species (ROS) by physical exercise is well documented in both animal and human studies. ROS are physiological products of aerobic metabolism, and impact on variety of metabolic processes including gene expression, protein turnover, inflammatory reaction, arachidonic acid immobilization, and cellular differentiation. ROS are released from muscle, endothelial, and immune cells to stimulate an adaptation to intense physical exercise. This adaptation includes changes in the immune and energetic status related to the cytokine synthesis such as tumor necrosis factor $\alpha$ (TNF $\alpha$ ) and interleukin-6 (IL-6) (Kosmidou et al. 2002, Valko et al. 2007, Radak et al. 2008).

Glutathione ( $\gamma$-glutamyl-cysteinyl-glycine) is the major non-protein thiol and one of the ROS sensors engaged in the cellular control of thiol redox. The ratio of reduced (GSH) to oxidized glutathione (GSSG) plays an essential role in regulating thiol-depend signal transduction. Changes in thiol redox affect transcriptional activity via activation or inhibition of transcriptional factors, thus modulating the expression of many genes such as TNF $\alpha$ and IL-6 genes (Allen and Tresini 2000, Ji et al. 2006).

Previously, it was hypothesized that the exercise-induced increases in TNF $\alpha$ and IL-6 are a consequence of an immune response to local damage in the working muscles (Nieman et al. 1998). However, Plomgaard et al. (2005) demonstrated that immune cells are not the source of the plasma TNF $\alpha$ and IL-6 during 
Table 1. Exercise intensity during training periods.

\begin{tabular}{|c|c|c|c|}
\hline Training period & Energetic area & Exercise load \% & Lactate $\mathrm{mmol} \cdot \mathrm{I}^{-1}$ \\
\hline \multirow{5}{*}{$\begin{array}{l}\text { Preparatory period } \\
\text { November, } 20 \text { days }\end{array}$} & Supporting (aerobic) & 19 & $<2$ \\
\hline & Aerobic & 21 & $2-4$ \\
\hline & Aerobic-anaerobic & 40 & $4-8$ \\
\hline & Anaerobic-lactate & 17 & $>8$ \\
\hline & Anaerobic-alactate* & 3 & $<5-6$ \\
\hline \multirow{5}{*}{$\begin{array}{l}\text { Preparatory period } \\
\text { December, } 25 \text { days }\end{array}$} & Supporting (aerobic) & 17 & $<2$ \\
\hline & Aerobic & 25 & $2-4$ \\
\hline & Aerobic-anaerobic & 42 & $4-8$ \\
\hline & Anaerobic-lactate & 13 & $>8$ \\
\hline & Anaerobic-alactate* & 3 & $<5-6$ \\
\hline \multirow{5}{*}{$\begin{array}{l}\text { Play-off round } \\
\text { April, } 20 \text { days }\end{array}$} & Supporting (aerobic) & 15 & $<2$ \\
\hline & Aerobic & 18 & $2-4$ \\
\hline & Aerobic-anaerobic & 46 & $4-8$ \\
\hline & Anaerobic-lactate & 17 & $>8$ \\
\hline & Anaerobic-alactate* & 4 & $<5-6$ \\
\hline \multirow{5}{*}{$\begin{array}{l}\text { Play-off round } \\
\text { May, } 20 \text { days }\end{array}$} & Supporting (aerobic) & 13 & $<2$ \\
\hline & Aerobic & 20 & $2-4$ \\
\hline & Aerobic-anaerobic & 49 & $4-8$ \\
\hline & Anaerobic-lactate & 12 & $>8$ \\
\hline & Anaerobic-alactate* & 6 & $<5-6$ \\
\hline
\end{tabular}

* short duration work (<10 s).

intense exercise. TNF $\alpha$ and IL-6 are expressed in human skeletal muscle in a strict fiber type specific fashion. TNF $\alpha$ is expressed by fast-twitch fibers whereas the expression of IL-6 is more prominent in slow-twitch fibers (Plomgaard et al. 2005). TNF $\alpha$ is a proinflammatory cytokine and increases primarily after isometric and eccentric exercises. IL-6 is an antiinflammatory cytokine and dramatically increases after long-term endurance exercises (Suzuki et al. 2002). High levels of TNF $\alpha$ impair glucose uptake by skeletal muscle, while IL-6 ensures glucose homeostasis and intramuscular glycogen stores (Steensberg et al. 2002, Petersen and Pedersen 2005). However, both cytokines play a very important role in muscle reconstruction after strenuous exercise and in the development of tolerance to ROS-induced muscle damage (Steensberg et al. 2002). TNF $\alpha$ initiates the breakdown of damaged muscle tissue, and IL- 6 stimulates the proliferation and differentiation of satellite cells (Peake et al. 2005).

Numerous studies explored the effects of a single physical effort on pro- and anti-inflammatory cytokines (Suzuki et al. 2002). However, little information is available on the inflammatory response to physical training and its relationship with ROS production and thiol redox status in athletes (Gokhale et al. 2007, Huffman et al. 2008, Rämson et al. 2008, Rong et al. 2008). Therefore, we attempted to evaluate the changes in plasma markers of ROS activity, thiol redox status, and inflammatory mediators including IL-6 and TNF $\alpha$, and then to demonstrate the association between cytokine production and thiol redox status in professional training activity.

\section{Methods}

Sixteen professional players of the Polish Basketball Extraleague (age 26.1 \pm 3.2 years, height $198.3 \pm 10.2 \mathrm{~cm}$, body mass $98.2 \pm 4.1 \mathrm{~kg}$ ) volunteered for this study; all participants gave informed consent. The protocol of the study was approved by the local ethics committee in accordance with the Helsinki Declaration. The subjects participated professionally in sport activities for an average 12.5 \pm 2.8 years. Markers of ROS activity, thiol redox status, cytokines, and creatine kinase activity were measured during the preparatory period (November, 
Table 2. Levels of plasma lipid peroxidation (TBARS) and protein carbonyls (PC) products, reduced and oxidised glutathione (GSH, GSSG), tumour necrosis factor $\alpha$ (TNF $\alpha$ ), interleukin-6 (IL-6) and creatine kinase (CK).

\begin{tabular}{llccccc}
\hline & \multicolumn{3}{c}{ Preparatory period } & \multicolumn{3}{c}{ Play-off round } \\
\cline { 2 - 7 } & November & December & Nov vs. Dec & April & May & Apr vs. May \\
\hline TBARS $\left(\mu \mathrm{mol} \cdot l^{-1}\right)$ & $1.88 \pm 0.39^{\text {ac }}$ & $0.89 \pm 0.14^{\text {bc }}$ & $P<0.001$ & $1.49 \pm 0.25$ & $0.98 \pm 0.20^{\text {ad }}$ & $P<0.001$ \\
$P C\left(\mathrm{nmol} \cdot \mathrm{mg}^{-1}\right)$ & $1.07 \pm 0.08$ & $0.80 \pm 0.12^{\text {bc }}$ & $P<0.001$ & $1.11 \pm 0.12$ & $0.89 \pm 0.16^{\text {ad }}$ & $P<0.01$ \\
GSH $\left(\mu \mathrm{mol} \cdot l^{-1}\right)$ & $2.73 \pm 0.55^{\text {ac }}$ & $1.77 \pm 0.49^{\text {bc }}$ & $P<0.001$ & $3.81 \pm 0.36$ & $1.45 \pm 0.51^{\text {ad }}$ & $P<0.001$ \\
$G S S G\left(\mu \mathrm{mol} \cdot l^{-1}\right)$ & $0.07 \pm 0.02$ & $0.07 \pm 0.01^{\text {bd }}$ & ns & $0.07 \pm 0.02$ & $0.05 \pm 0.01^{\text {ad }}$ & $P<0.001$ \\
$I L-6\left(n g \cdot l^{-1}\right)$ & $1.31 \pm 0.35^{\text {ac }}$ & $0.82 \pm 0.20^{\text {bc }}$ & $P<0.001$ & $0.53 \pm 0.23$ & $0.73 \pm 0.23^{\text {ad }}$ & $\mathrm{ns}$ \\
$T N F \alpha\left(n g \cdot l^{-1}\right)$ & $0.87 \pm 0.26$ & $0.85 \pm 0.19^{\text {bd }}$ & ns & $0.99 \pm 0.34$ & $1.43 \pm 0.34^{\text {ad }}$ & $P<0.01$ \\
$C K\left(U \cdot l^{-1}\right)$ & $238 \pm 74$ & $186 \pm 45^{\text {bd }}$ & ns & $261 \pm 147$ & $327 \pm 141$ & ns \\
\hline
\end{tabular}

Double letters indicate statistically significant differences $(P<0.01)$ between preparatory period and play-off round: ${ }^{\text {ac }}$ Nov $v s$. Apr,

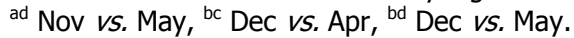

December) and during the play-off round (April, May). All subjects underwent the same, standardized training program. Details of the training cycles reported by the coach are shown in Table 1. During the study subjects were asked to avoid drugs or nutrition supplement that could interfere with pro-antioxidant evaluation.

Each subject was asked to avoid physical effort for $24 \mathrm{~h}$ before the laboratory measurements. Blood samples were taken from an antecubital vein with an anticoagulant (EDTA-K $\mathrm{K}_{2}$ ) in the morning (8 a.m.) after $15 \mathrm{~min}$ of rest. After collection the samples were immediately placed in $4{ }^{\circ} \mathrm{C}$ temperature. Within $10 \mathrm{~min}$, they were centrifuged at $2500 \mathrm{~g}$ and $4{ }^{\circ} \mathrm{C}$ for $10 \mathrm{~min}$. Aliquots of plasma were stored at $-20^{\circ} \mathrm{C}$. All assays were performed within 5 days of plasma collection.

Measurement of thiobarbituric acid reactive substance (TBARS) level, according to the method of Buege and Aust (1991), was used to estimate plasma lipid peroxidation products. To avoid further peroxidation, plasma samples were treated with $15 \%$ trichloroacetic acid containing $0.25 \mathrm{M}$ hydrochloric acid. TBARS level was expressed as nmol of malondialdehyde using 1,1,3,3tetraethoxypropane as a standard. The TBARS detection limit was $0.13 \mathrm{nmol} \cdot \mathrm{ml}^{-1}$.

Plasma protein carbonyls (PC) were measured by the method of Levine et al. (1990) using 2,4-dinitrophenyl hydrazine. The carbonyl content was calculated using an extinction coefficient of $22000 \mathrm{M}^{-1} \mathrm{l}^{-1 .} \mathrm{cm}^{-1}$ and expressed as nmol PC per mg of plasma protein. Protein concentration was determined by the method of Bradford (1976). The intra-assay coefficient of variation (CV) was $<10 \%$ for $\mathrm{PC}$ and TBARS procedures.
Plasma reduced (GSH) and oxidized glutathione (GSSG) were measured with Oxis Research kit (USA). The concentrations of GSH and GSSG were calculated using reduced glutathione as a standard and the results were expressed in $\mu \mathrm{mol}{ }^{-1} 1^{-1}$. Detection limits for the GSH and GSSG were $0.1 \mu \mathrm{mol} \cdot \mathrm{l}^{-1}$ and $0.02 \mu \mathrm{mol} \cdot \mathrm{l}^{-1}$, respectively. The intra-assay coefficients of variation (CV) for GSH and GSSG were $0.96 \%$ and $6.45 \%$, respectively. Thiol redox status was calculated according to the following equation: ( $\left.\mathrm{GSH}_{\text {total }}-2 \mathrm{GSSG}\right) / \mathrm{GSSG}$.

Plasma tumor necrosis factor (TNF $\alpha$ ) and interleukin-6 (IL-6) levels were determined by enzyme immunoassay methods using commercial kits (R\&D Systems, USA). Detection limits for TNF $\alpha$ and IL-6 were $0.038 \mathrm{pg}^{\cdot} \mathrm{ml}^{-1}$ and $0.04 \mathrm{pg}^{\prime} \mathrm{ml}^{-1}$, respectively. The intraassay coefficient of variation (CV) was $<8.0 \%$ for both cytokines.

Creatine kinase (CK) activity was evaluated using the diagnostic assays for the kinetic enzyme analyzer Konelap 60 BioMerieux (France). CK detection limit for the applied kit was $6 \mathrm{U} \cdot \mathrm{I}^{-1}$. The intra-assay coefficient of variation (CV) for the CK kit was $1.85 \%$.

Lactate concentration in the capillary blood was assessed before and 5 min after training using LKM 140 Dr Lange kit (Germany). The intra-assay coefficient of variation $(\mathrm{CV})$ for the lactate kit was $2.5 \%$.

Statistical calculations were performed using STATISTICA 8.0. Statistical significance was assessed by one-way analysis of variance (ANOVA) and Tukey post-hoc test. Associations among measured parameters were analyzed using Pearson's linear regression (coefficient, $r$ ). Statistical significance was set at $P<0.05$. Results are expressed as mean \pm S.D. 


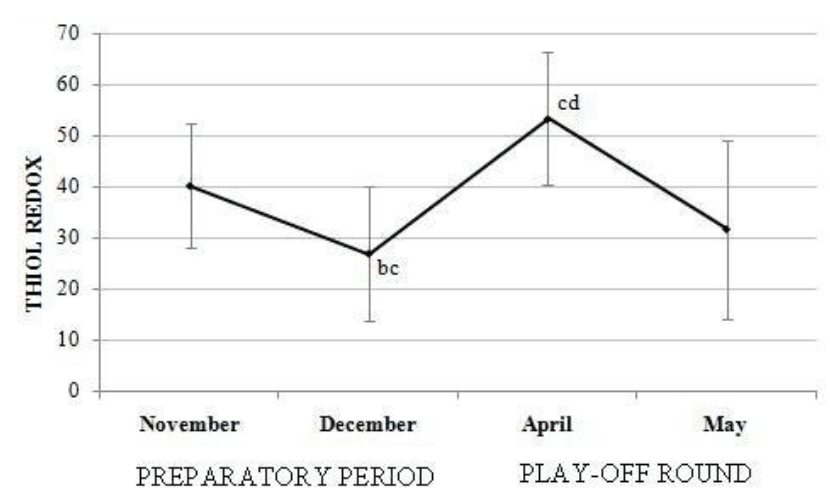

Fig. 1. Changes in thiol redox status (GSHtotal-2GSSG/GSSG) in basketball players in training periods; double letters indicate statistically significant differences $(P<0.001)$ between cycles of the preparatory period and play-off round: bc Dec vs. Apr, cd Apr vs. May.

\section{Results}

The analysis of ROS activity markers, thiol redox status, and cytokine levels showed changes dependent on exercise load in particular training cycles (Tables 1 and 2).

TBARS concentration was significantly elevated in the preparatory period compared with the play-off round. The highest level of TBARS was found at the beginning of the preparatory period (November). PC did not demonstrate the same degree of variability between training periods. However, the measurement of ROS activity markers showed consistency over time, i.e. TBARS and PC were elevated at the beginning of analysed periods (November, April), and dropped at the end of training periods (December, May).

Changes in GSH were similar to TBARS and PC, i.e. GSH increased in November and April and decreased in December and May. The highest value of GSH was observed at the beginning of the play-off round. GSSG concentration did not demonstrate significant changes in the preparatory period, but increased during the play-off period. Both glutathione forms were significantly higher in April than in May.

Thiol redox status, calculated from GSH and GSSG ratio, was lowest in December (26.9 \pm 13.3 $\left.\mu \mathrm{mol} \cdot \mathrm{l}^{-1}\right)$ and highest in April $\left(53.3 \pm 13.0 \mu \mathrm{mol} \cdot \mathrm{l}^{-1}\right)$ (Fig. 1). Thiol redox directly correlated with TBARS and PC in both training cycles (Tables 3 and 4).

Cytokines responded differently to exercise loads during the analyzed training cycles (Table 2). IL-6 concentration was significantly elevated in the preparatory period and reached the highest level in November. Opposite changes were observed in TNF $\alpha$ concentration: low levels were observed in the preparatory period and high levels in game season. TNF $\alpha$ reached the highest level at the end of the play-off round (May), corresponding to performance of anaerobicalactate efforts.

IL-6 directly correlated with thiol redox status $(\mathrm{r}=0.423)$ in the preparatory period whereas TNF $\alpha$ inversely correlated with thiol redox $(r=-0.509)$ in the play-off round (Tables 3 and 4).

Table 3. Relationships (correlation coefficients) between thiol redox status, cytokines (IL- 6 and TNF $\alpha$ ), and oxidative damage markers (TBARS, PC) in the preparatory period.

\begin{tabular}{lllll}
\hline & IL-6 & TNF $\alpha$ & TBARS & PC \\
\hline Thiol redox & $0.423^{*}$ & -0.147 & $0.415^{*}$ & $0.683^{*}$ \\
\hline
\end{tabular}

$* P<0.05$.

Table 4. Relationships (correlation coefficients) between thiol redox status, cytokines (IL- 6 and TNF $\alpha$ ), and oxidative damage markers (TBARS, PC) in the play-off round.

\begin{tabular}{ccccc}
\hline & IL-6 & TNF $\alpha$ & TBARS & PC \\
\hline Thiol redox & -0.159 & $-0.509 *$ & $0.572 *$ & $0.481^{*}$ \\
\hline
\end{tabular}

$* P<0.05$.

CK activity as a marker of muscle damage was the highest in May, similar to TNF $\alpha$. However, there were no significant correlations between CK and TNF $\alpha$ in the play-off round.

\section{Discussion}

The main purpose of this study was to determine the role of thiol redox as a second messenger between ROS generation and cytokine response during training activity.

Thiobarbituric acid and dinitrophenyl hydrazine, for the detection of lipid peroxidation and protein carbonylation products, are the most widely used markers of ROS activity. Although plasma TBARS and PC are non-specific techniques, they adequately express the complex process of peroxidation and carbonylation followed by single or repeated exercises (Margaritis et al. 1997, Allesio et al. 2000, Metin et al. 2003, Knez et al. 2007). The present study demonstrated that the changes in lipid peroxidation and protein carbonylation in basketball players were specifically dependent on exercise loads applied during the investigated periods. 
Both TBARS and PC were elevated at the beginning of preparatory and play-off periods, and values dropped at the end of training periods, showing inverse relationship to the percent of aerobic efforts. This suggests that exercises performed at the aerobic level enhanced antioxidant defense activity and thus reduced ROS generation in athletes. The present results partially confirm those obtained in previous studies. The typical aerobic endurance exercise such as triathlon racing and cycling training did not induce lipid peroxidation and even decreased resting concentration of peroxidation products (Mena et al. 1991, Margaritis et al. 1996, Knez et al. 2007). Oztasan et al. (2004) and Brooks et al. (2008) established that high-volume endurance training effectively prevents acute exhaustive exercise-induced ROS generation by upregulation of the antioxidant system.

It is well known that ROS serve as messengers, activating adaptive responses by thiol redox-sensitive signaling pathways (Allen and Tresini 2000, Ji et al. 2006). In our study, markers of ROS activity directly correlated with thiol redox status in both training periods, demonstrating the involvement of ROS in thiol redox disturbance. Thiol status, i.e. reduced and oxidized glutathione ratio, has been favorably linked to two redoxsensitive transcription factors, nuclear factor $\kappa \mathrm{B}(\mathrm{NK}-\kappa \mathrm{B})$ and activator protein-1 (AP-1). Expression of these transcription factors may be induced by extremely high or low levels of reduced or oxidized glutathione, resulting in increased IL-6 and TNF $\alpha$ expression in skeletal muscle. In addition, TNF $\alpha$ is known to be a potent activator of NF- $\mathrm{KB}$ in immune cells (Kersick and Willoughby 2005).

The cytokine response to physical exercise has been studied by several authors in recent years (Steensberg et al. 2002, Suzuki et al. 2002, Plomgaard et al. 2005, Steinberg et al. 2007). Petersen and Pedersen (2005) reported that plasma IL-6 and TNF $\alpha$ increase exponentially during physical effort in relation to exercise intensity, duration, mass of working muscles, and individual endurance capacity. It was also demonstrated that mRNA for IL- 6 and TNF $\alpha$ are upregulated in contracting skeletal muscle, and that the transcriptional rate of IL- 6 and TNF $\alpha$ genes are markedly enhanced by ROS generation (Kosmidou et al. 2002, Plomgaard et al. 2005).

Our results show that professional sport training significantly influenced plasma cytokine levels. IL-6 was higher in the preparatory period than in the play-off round. According to Petersen and Pedersen (2005) IL-6 acts in a hormone-like manner to maintain glucose homeostasis, induce lipolysis and fat oxidation, exert an inhibitory effect on TNF $\alpha$ production, and stimulate the proliferation of satellite cells and differentiation of myoblasts. Thus, it seems that an increase of IL- 6 is important for the development of optimal adaptation to intense physical exercise. The decline in IL-6, observed in the game season, may be related to muscle lactate accumulation during competition (McInnes et al. 1995). The high lactate produced during intense exercise likely impairs the ability of muscle cells to produce IL-6 (Suzuki et al. 2002).

$\mathrm{TNF} \alpha$ increased during the play-off round and reached the highest level in May when muscle structure was disrupted, as expressed by high CK activity. Muscle damage attracts neutrophils and macrophages to the site of injury, which contribute to the degradation of damaged tissue by releasing pro-inflammatory cytokines such as TNF $\alpha$ (Peake et al. 2005). Therefore, an increase in plasma TNF $\alpha$ may be a useful index of phagocytosis and reconstruction of muscle after intense exercise.

An important finding of our study was an integration of ROS production and cytokine response with thiol redox status. Thiol redox correlated with markers of ROS activity in both training periods. However, the relationship between thiol redox and cytokines was markedly dependent on training loads. In the preparatory period, thiol redox positively correlated with the high levels of IL-6, whereas during the play-off round, it was inversely correlated with the elevated TNF $\alpha$ levels.

In conclusion, our study demonstrates that 1) thiol redox status plays an important role in IL-6 and $\mathrm{TNF} \alpha$ release in physical training activity, 2) IL-6 dominates during the preparatory period, whereas TNF $\alpha$ is the predominant cytokine in the play-off round, and 3) determination of selected cytokine might be introduced for the metabolic evaluation of training periods.

\section{Conflict of Interest}

There is no conflict of interest.

\section{Acknowledgements}

This work was supported by grant for research from University of Physical Education Poznan, Poland. 


\section{References}

ALLEN GR, TRESINI M: Oxidative stress and gene regulation. Free Radic Biol Med 28: 463-499, 2000.

ALESSIO HM, HAGERMAN AE, FULKERSON BK, AMBROSE J, RICE RE, WILEY RL: Generation of reactive oxygen species after exhaustive aerobic and isometric exercise. Med Sci Sports Exerc 32: 1576-1581, 2000.

BRADFORD MM: A rapid and sensitive method for the quantitation of microgram quantities of protein utilizing the principle of protein - dye binding. Anal Biochem 72: 248-254, 1976.

BUEGE J, AUST SD: The thiobarbituric acid assay. In: Techniques in Free Radical Research. CA RICE-EVANS, AT DIPLOCK, MCR SYMONS (eds), Elsevier, Amsterdam, 1991, pp 147-148.

BROOKS SV, VASILAKI A, LARKIN LM, MCARDLE A, JACKSON MJ: Repeated bouts of aerobic exercise lead to reductions in skeletal muscle free radical generation and nuclear factor kappaB activation. J Physiol Lond 586: 3979-3990, 2008.

GOKHALE R, CHNADRASHEKARA S, VASANTAKUMAR KC: Cytokine response to strenuous exercise in athletes and non-athletes-an adaptive response. Cytokine 40: 123-127, 2007.

HUFFMAN KM, SLENTZ CA, BALES CW, HOUMARD JA, KRAUS WE: Relationships between adipose tissue and cytokine responses to a randomized controlled exercise training intervention. Metabolism 57: 577-583, 2008.

JI LL, GOMEZ-CABRERA MC, VINA J: Exercise and hormesis: activation of cellular antioxidant signaling pathway. Ann NY Acad Sci 1067: 425-435, 2006.

KERKSICK C, WILLOUGHBY D: The antioxidant role of glutathione and N-acetyl-cysteine supplements and exercise-induced oxidative stress. J Int Soc Sports Nutr 2: 38-44, 2005.

KNEZ WL, JENKINS DG, COOMBES JS: Oxidative stress in half and full Ironman Triathletes. Med Sci Sports Exerc 39: 283-288, 2007.

KOSMIDOU I, VASSILAKOPOULOUS T, XAGORARI A, ZAKYNTHINOS S, PAPAPETROPOULOS A, ROUSSOS C: Production of interleukin-6 by skeletal myotubes. Role of reactive oxygen species. Am J Respir Cell Mol Biol 26: 587-593, 2002.

LEVINE RL, GARLAND D, OLIVER CN: Determination of carbonyl content in oxidatively modified proteins. Methods Enzymol 186: 464-479, 1990.

MARGARITIS I, TESSIER F, ROCHARD MJ, MARCONNET P: No evidence of oxidative stress after a triathlon race in highly trained competitors. Int J Sports Med 18: 186-190, 1997.

MCINNES SE, CARLSON JS, JONES CJ, MCKENNA MJ: The physiological load imposed on basketball players during competition. $J$ Sports Sci 13: 387-397, 1995.

MENA P, MAYNAR M, GUTIERREZ JM, MAYNAR T, TIMON J, CAMPILLO JE: Erythrocyte free radical scavenger enzymes in bicycle professional racers. Adaptation to training. Int J Sports Med 12: 563-566, 1991.

METIN G, GÜMÜSTAS K, USLU E, BELCE A, KAYSERILIOGLU A: Effect of regular training on plasma thiols, malondialdehyde and carnityne concentrations in young soccer players. Chin J Physiol 46: 35-39, 2003.

NIEMAN DC, NEHLSEN-CANNARELLA SL, FAGOAGA OR, HENSON DA, UTTER A, DAVIS JM, WILLIAMS F, BUTTERWORTH DE: Influence of mode and carbohydrate on the cytokine response to heavy exertion. Med Sci Sports Exerc 30: 671-678, 1998.

OZTASAN N, TAVSI S, GUMUSTEKIN K, ALTINKAYNAK K, AKTAS O, TIMUR H, SIKTAR E, KELES S, AKAR S, AKCAY F, DANE S, GUL M: Endurance training attenuates exercise-induced oxidative stress in erythrocytes in rat. Eur J Appl Physiol 91: 622-627, 2004.

PEAKE JM, SUZUKI K, WILSON G, HORDERN M, NOSAKA K, MCKINNON L, COOMBES J: Exercise-induced muscle damage, plasma cytokines, and markers of neutrophil activation. Med Sci Sports Exerc 37: 737-745, 2005.

PETERSEN AMW, PEDERSEN BK: Anti-inflammatory effect of exercise. J Appl Physiol 98: 1154-1162, 2005.

PLOMGAARD P, PENKOVA M, PEDERSEN BK: Fiber type specific expression of TNF-alpha, IL-6 and IL-18 in human skeletal muscles. Exerc Immunol Rev 11: 53-64, 2005.

RADAK Z, CHUNG HY, GOTO S: Systemic adaptation to oxidative challenge induced by regular exercise. Free Radic Biol Med 44: 153-159, 2008. 
RÄMSON R, JÜRIMÄE J, JÜRIMÄE T, MÄESTU J: The influence of increased training volume on cytokines and ghrelin concentration in college level male rowers. Eur J Appl Physiol 104: 839-846, 2008.

RONG C, BEI H, YUN M, YUZHU W, MINGWU Z: Lung function and cytokine levels in professional athletes. J Asthma 45: 343-348, 2008.

STEENSBERG A, HALL G, OSADA T, SACCHETTI M, SALTIN B, PEDERSEN BK: Production of interleukin-6 in contracting human skeletal muscles can account for the exercise-induced increase in plasma interleukin-6. J Physiol Lond 529: 237-242, 2000.

SUZUKI K, KAKAJI S, YAMADA M, TOTSUKA M, SATO K, SUGAWARA K: Systemic inflammatory response to exhaustive exercise. Cytokine kinetics. Exerc Immunol Rev 8: 46-48, 2002.

VALKO M, LEIBFRITZ D, MONCOL J, CRONIN MTD, MAZUR M, TESLER J: Free radicals and antioxidants in normal physiological functions and human disease. Int J Biochem Cell Biol 39: 44-84, 2007. 